The final publication is available at Elsevier via https://dx.doi.org/10.1016/j.jwpe.2018.09.009 ( 2018. This manuscript version is made available under the CC-BY-NC-ND 4.0 license https://creativecommons.org/licenses/by-nc-nd/4.0/

\title{
Performance of Polyacrylamide and Poly(acrylamide/sodium acrylate) Hydrogel-Coated Mesh for Separation of Oil/Water Mixtures
}

\author{
Chandra Mouli R. Madhuranthakam ${ }^{1}$, Amal Alsubaei ${ }^{2}$, Ali Elkamel ${ }^{3,4}$ \\ 1 - Chemical Engineering Department, Abu Dhabi University, P.O.Box. 59911, Abu Dhabi,UAE \\ 2,3 - Chemical Engineering Department, University of Waterloo, Waterloo, Canada, N2L 3G1 \\ 4 - Chemical Engineering Department, Khalifa University, Abu Dhabi, UAE
}

\begin{abstract}
Hydrogels are widely used as coagulant in water treatment in addition to using in tissue engineering due to their high water absorption capacity and biocompatibility. Our research objective is to synthesize a reinforced membrane made using hydrogels targeted for the separation of water from oil-water mixtures. The reinforced hydrogel is synthesized by graft polymerization of water-soluble polymer such as polyacrylamide (PAM) homo-polymer and poly (Na-Ac/AM) copolymer reinforcing on a thin-fine metallic mesh. Different factors such as the mesh size, the percentage of coated-hydrogel, swelling index are varied to study their effect on the separation efficiency. The morphology of the reinforced hydrogel was analyzed by using scanning electron microscopy. In the case of hydrogels made from copolymers, the effect of the corresponding concentration of the copolymer on the separation efficiency is studied. Oil-water mixtures of 5/95 and 10/90 oil/water \% were chosen based on industrial practices as it is not typical to find a wastewater stream with more than $30 \%$ oil composition. The wettability of water and oil on the PAM- hydrogel coated mesh was evaluated using the contact angle measurements obtained at ambient temperature. It was found that PAM polymer and Na-Ac/AM copolymer hydrogel coated meshes is a super-hydrophilic in an air-solid-liquid three phases with both the contact angle of oil and water less than $15^{\circ}$. Underwater contact angles for the four different sizes of meshes based on PAM coated mesh are observed to be greater than $90^{\circ}$. This indicates the oleophobic properties of the coated mesh under the water and oil cannot penetrate through the coated mesh while the water alone is absorbed through it. The difference in the water flow or separation time becomes smaller with increasing the mesh's pore size up to 80 micron, while separation time was higher for 200 micron mesh as more hydrogel was blocking the pores. In contrast, the separation time is faster with the copolymer due to the higher swelling capacity of the copolymer. Therefore, under-water oleo-phobic properties of the PAM coated mesh make it a promising candidate for separation of oil/water emulsions with more than $95 \%$ separation efficiency.
\end{abstract}

Keywords: acrylamide, hydrogel, oil/water separation, reinforced membrane, graft polymerization

*Corresponding Author: email: chandra.mouli@adu.ac.ae

Ph: +971 25015304 
Highlights

- $\quad$ stainless steel mesh reinforced hydrogel membrane

- separation of oil/water emulsions

- characterization in terms of morphology, contact angle were studied

- $\quad$ polyacrylamide (PAM) homo-polymer and poly (Na-Ac/AM) copolymer membranes

- effect of pore size, concentration of polymers and oil/water were studied.

Graphical Abstract
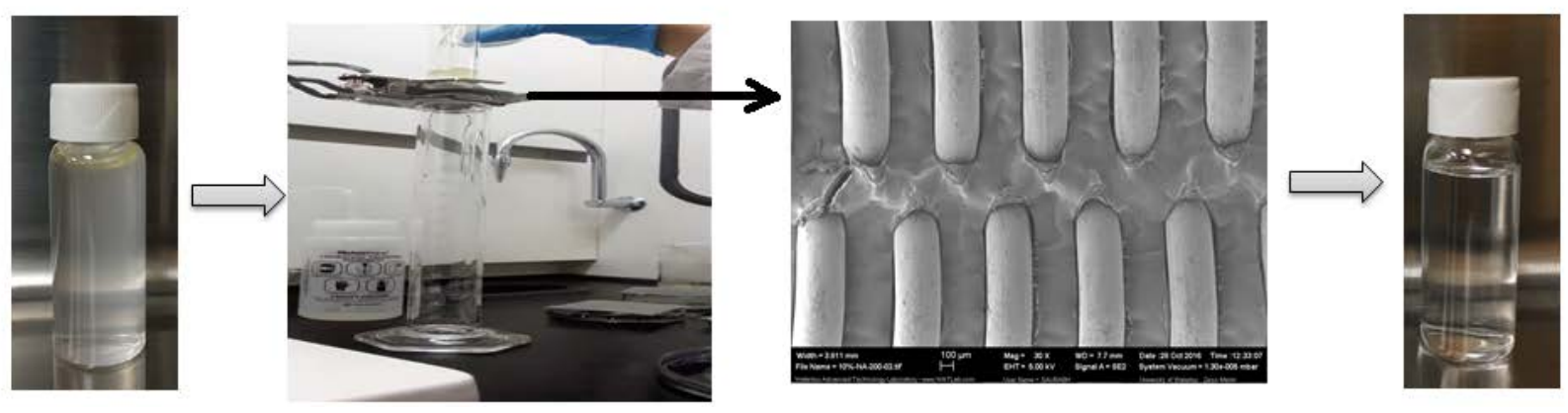


\section{Introduction}

It is estimated that 20 billion barrels of wastewater per year are produced by the oil and gas industry in the US alone [1,2]. Oil/water separation is an important issue that many industries have to address not only to avoid wastage but also meet environmental regulations. In addition to the existing conventional methods, new innovative techniques which are more efficient are needed to overcome this challenge. The objective of this research article is to design and synthesize hydrogels targeted for separation of water from oil/water mixture. Hydrogels describes three-dimensional network structures which are obtained from synthetic or natural polymers and are defined as cross-linked polymeric materials that can absorb significant amounts of water by swelling within its structure yet, they are not soluble in water $[3,4]$. The degree of crosslinking which is related to the branching in a polymer has significant effect on the thermal and mechanical properties. Water-soluble oleophobic polymers such as polyacrylamide have many industrial applications such as in the production of paints, cosmetics, oil recovery and drilling fluids due to the hydrophobic alkyl and aryl groups distributed along the polymer chains which enhances the thickening properties and air-liquid surface activities. While there are currently membrane-based technologies that can be used to separate oil/water mixtures they often have drawbacks, such as requiring high-energy inputs or expensive equipment [5, 6]. An appealing technology that could be used to meet this demand is to use reinforced hydrogels. The reinforcement usually gives the extra support for the hydrogel to withstand pressure exerted by the fluid on one side. For the application of treating produced water, these hydrogels can be engineered to be oleo-phobic and hydrophilic. The hydrophilicity allows water to easily pass through the hydrogel, while the oleophobicity prevents oil from entering or passing through the hydrogel. In this research article, we use polyacrylamide (PAM) based hydrogels to achieve the objective of separating oil from oil/water mixture. A major benefit of using PAM based reinforced membrane is that the fouling of the hydrogel by the oil is less. Other benefits include the remnant oil concentration in the filtered water being very low and it is very economical to synthesize these reinforced hydrogels. Mohana et al. [7] has investigated superabsorbent polymer (SAP), where the preparation is based on using acrylamide, acrylic acid, and its salts by inversesuspension polymerization and diluted solution polymerization. These superabsorbent polymers has the ability to absorb water or aqueous solutions up to hundreds of times of their original weight and has been used in personal hygiene products $[8,9]$. The swelling capacity of SAPs 
increases with increasing crosslink density, which reaches a maximum at an optimum crosslink density, and then decreases as the network becomes denser [10-12]. Moreover, it was stated that with at low $\mathrm{pH}$, the swelling ratio of $\mathrm{Na}-\mathrm{Ac} / \mathrm{AM}$ copolymer decrease as the sodium carboxylate group on the polymer are protonated and hydrogel shrink and become more hydrophobic. On the other hand, at high $\mathrm{pH}$, the swelling index decreases due to the effect of excess sodium ion in the swelling media, which blocks the carboxylate anion and prevents the effective of anion-anion repulsion. Therefore, for $\mathrm{pH}$ from 4 to 8 , some of the carboxylic acid groups are ionized and the electrostatic repulsion between them produces improvement of the swelling ratio [13]. We proposed to use hydrogels synthesized using acrylamide (AM) and sodium acrylate monomers (Na-Ac). For controlled crosslink density within the hydrogel, graft polymerization technique is used to make the reinforced hydrogel from these monomers. In the case of hydrogels with AM and Na-Ac monomers, a supposedly linear chain of AM will have attached to it side chains of $\mathrm{Na}$-Ac which contributes to a three dimensional network that varies with respect to the properties of the polymers formed from individual monomers alone. The corresponding effect of the concentration of these monomers on the separation efficiency is studied in this research project.

\section{Materials and Methods}

\subsection{Materials}

The homo-polymer Polyacrylamide (PAM) (average M.W. 5,000,000-6,000,000) was of laboratory grade in the form of powder. It was purchased from Poly Science, Inc. (Warrington, PA, USA). Acrylamide (AM), which is a white crystalline solid (with M.W. $71.08 \mathrm{~g} / \mathrm{mol}$ that is suitable for electrophoresis, >99\%, A8887-100G), Sodium Acrylate (Na-Ac), N, N' Dimethylene Bisacrylamide (BIS), (for electrophoresis, >99.5\%), and 2,2' Diethoxyacetophenone (DEOPS) (>95\%) were purchased from Sigma-Aldrich (Oakville, Ontario, Canada). Distilled water was used in all experiments. Stainless steel meshes were purchased from TWP Inc. (Berkeley, CA, USA) in five different sizes-25, 55,65,80 and 200 micron. Glass tubes were ordered from Chemglass Life Sciences (Vineland, NJ, USA), CG 124 with the pinch clamps, (ORing Joint, 25mm ID, 30mm Tube OD, O-Ring Size: 217, Clamp Size: 35). The oil used is canola oil which was purchased from a local store. Sodium sulphate and n-hexane used for extraction and drying were obtained from Sigma-Aldrich. 


\subsection{PAM hydrogel formulation}

The PAM hydrogel-coated meshes were prepared using a photo-initiated polymerization process with acrylamide (AM), N, N'-methylene bisacrylamide (BIS), 2,2'-diethoxyacetophenone (DEOP), polyacrylamide (PAM) as the precursor to the final product and a cross-linker. In the case of copolymer hydrogel, both acrylamide and sodium acrylate are used as monomers. BIS is an important factor in governing the size of the pores of the hydrogel which in turn affects the swelling index of the hydrogel. DEOP is a photo initiator, which forms radicals particularly when subjected to UV light. PAM, AM and BIS (0.5, (50,55,60), 1.5 wt. \% respectively) were dissolved in (47, 42 and 37 wt. \%) distilled water in the corresponding proportion in three $50 \mathrm{ml}$ glass beakers at room temperature to make three different polymer concentrations based on AM wt. \%. The water was first degassed with nitrogen gas for 15 minutes in order to remove any dissolved oxygen from it, which would act as an inhibitor that kills the polymerization, which is undesirable at this stage. The composition of the chemicals used in the synthesis of these hydrogels is summarized in Table 1. The resultant solution was stirred for 1 hour at $600 \mathrm{rpm}$. It was observed that with an increase in the monomer concentration, it takes a longer time to make a homogenous solution of all these ingredients. DEOP was added to the solution in the last and is mixed for 15 more minutes. Since DEOP is photosensitive, it was added in the last to avoid any early onset polymerization. For each batch, a total solution weight of $40.0 \mathrm{~g}$ was made, which was used for coating three stainless steel meshes. Once the chemicals were added, the solution was covered completely using an Aluminium foil to avoid any photo-initiation due to the day light. The same formulation was used for all of the experiments to produce three different hydrogel concentrations.

Table 1 Formulation for PAM Hydrogel Solution.

\begin{tabular}{|l|c|}
\hline Ingredient & \% Proportion (by weight) \\
\hline Acrylamide & $50 / 55 / 60$ \\
\hline Polyacrylamide & 0.5 \\
\hline N, N'-methylene Bis-acrylamide (BIS) & 1.5 \\
\hline 2,2'-diethoxyacetophenone (DEOP) & 1 \\
\hline DI-Water & $47 / 42 / 37$ \\
\hline
\end{tabular}

Table 2. Formulation for AM/Na-Ac copolymer solution

$$
\text { Ingredient }
$$

\% Proportion (by weight) 


\begin{tabular}{|c|c|}
\hline Acrylamide & $5 / 45$ \\
\hline Sodium Acrylate & $55 / 10$ \\
\hline Polyacrylamide & 0.5 \\
\hline N, N'-methylene bis-acrylamide (BIS) & 1.5 \\
\hline 2,2'-diethoxyacetophenone (DEOP) & 1 \\
\hline DI-Water & $37 / 42$ \\
\hline
\end{tabular}

Once the polymer or copolymer solution (obtained using formulation shown in Table 2) turned into a homogenous solution, it is then poured into a square dish for easy coating of the mesh pieces. The pre-cleaned mesh was then carefully immersed in the solution for about 5 seconds before it was drawn out slowly and horizontally with the solution adhered to the surface of the mesh, as shown in Figure 1. The mesh was removed horizontally and utmost care was taken decreasing the chance of the solution dripping off the mesh.

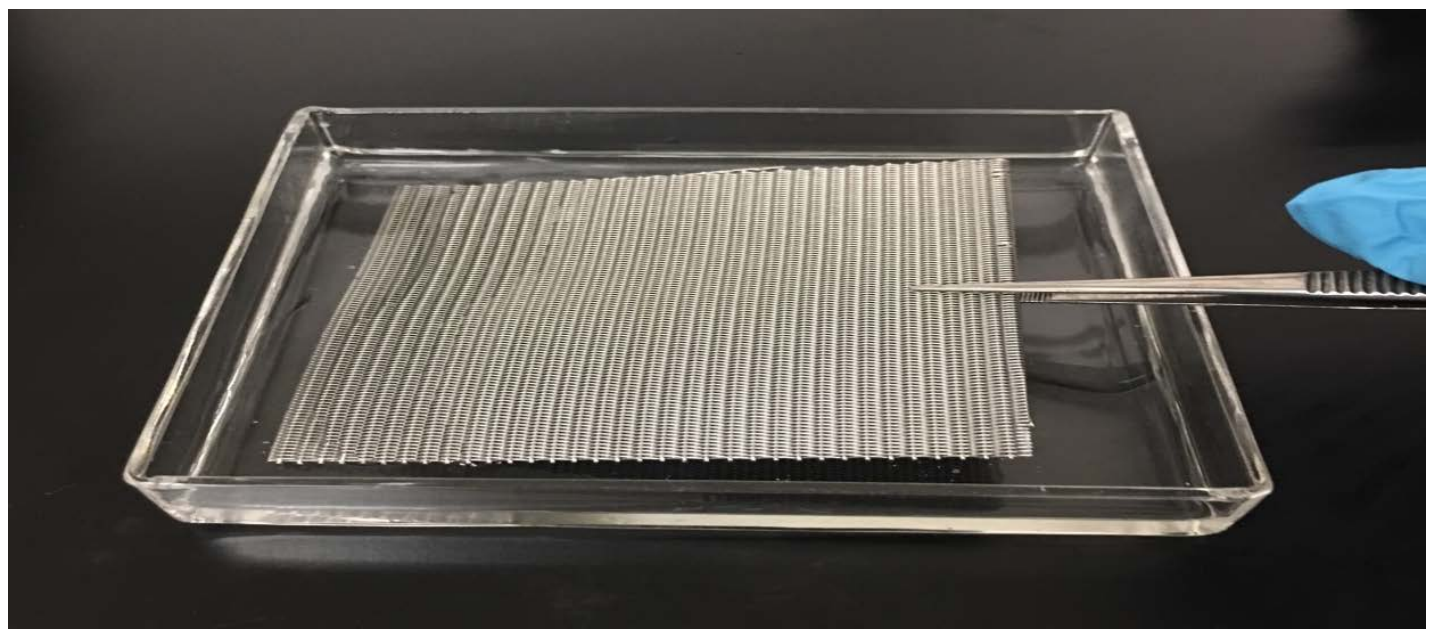

Figure 1. Dipping of a clean mesh horizontally in the solution before polymerization

After dipping, the mesh was irradiated by UV light (360 nm) for 90 min to fully polymerize the solution. This allowed photo-initiation of the coated solution in order to transform it into the polymerized hydrogel. The lamp height (measured from the top of coated mesh surface to lamp surface) was controlled at $5 \mathrm{~cm}$. After polymerization, the coated mesh was washed with distilled water. The same procedure was used for fabrication of PAM polymer and Na-Ac/AM copolymer meshes.

\subsection{Filtration Set up}


Oil water mixtures were prepared by mixing desired quantities of the two components in a beaker at $600 \mathrm{rpm}$ and 15 minutes in order to form a homogeneous mixture (as shown in Figure 2. a). The filtration set up consisted of two graduated glass cylinders which had groves to accommodate an O-ring that holds the reinforced membrane tightly without any leaks (as shown in Figure 2. b). Oil/water mixtures are poured in the top cylinder and water is collected in the bottom cylinder. The separation time was recorded for each experiment. The filtered oil form the oil/water mixture remained in the upper glass cylinder.
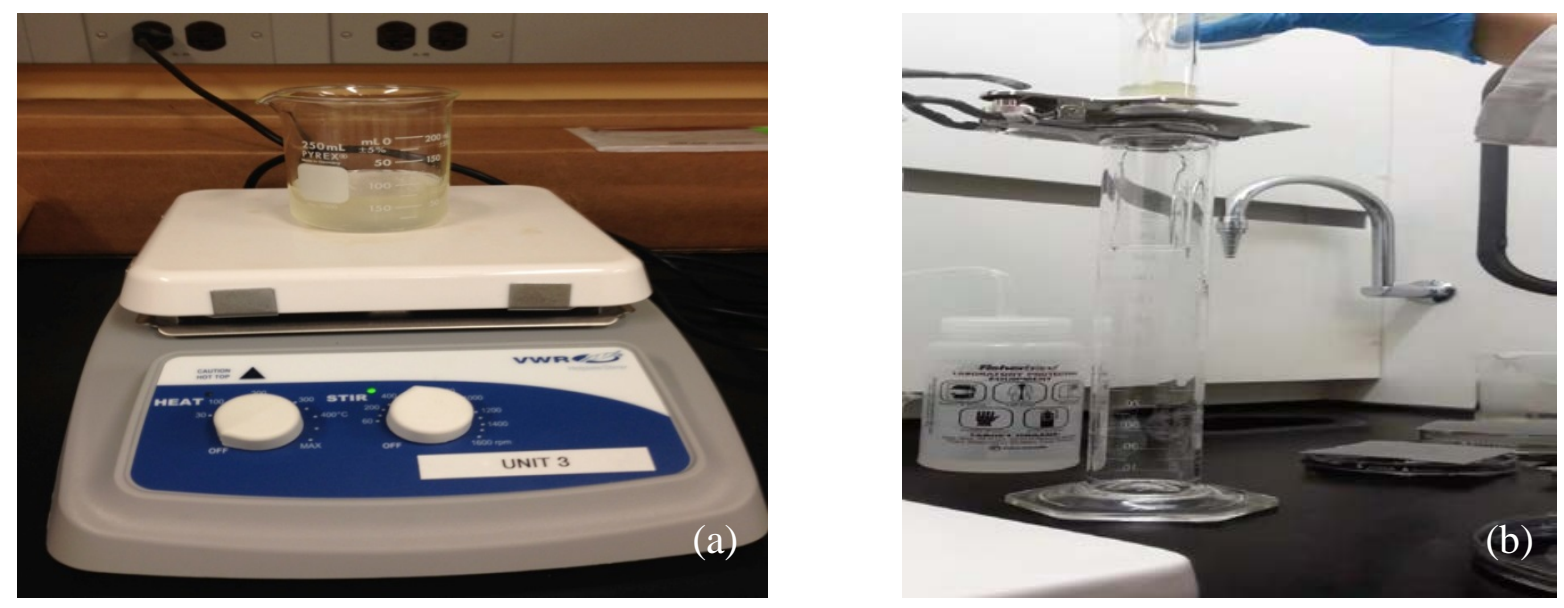

Figure 2 (a) Oil/water mixture during mixing and (b) separation process of oil/water mixture 2.4 Design of Experiments

The complete procedure of doing experiments is shown in Figure 3. Effect of the polymer and copolymer hydrogel on the separation time is studied by varying the mesh size. Four different pore sizes of the mesh, 55, 65, 80 and 200 micron, are used. As shown in Table 3.4 the oil/water mixture composition was varied at three levels. Oil weight percentages ranging from 5\% to 30\% are chosen to be tested for PAM and from 5 to $10 \%$ for Na-Ac/A copolymer. 
Experiment Feed Compositions:

- Deionized Water (DI water)

- Polyacrylamide (PAM)

- Acrylamide (AM)

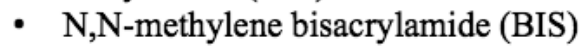

- 2,2'-diethoxy-acetophenone (DEOP)

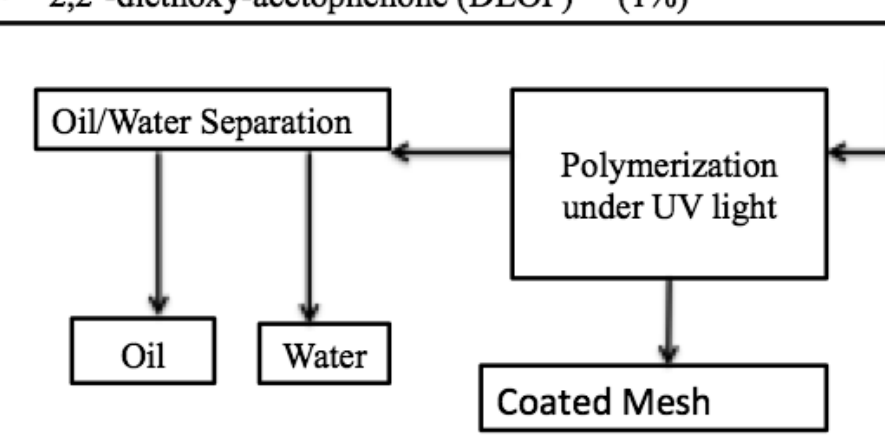

$(47,42,37 \%)$

$(0.5 \%)$

$(50,55,60 \%)$

$(1.5 \%)$

$(1 \%)$

Mesh coating

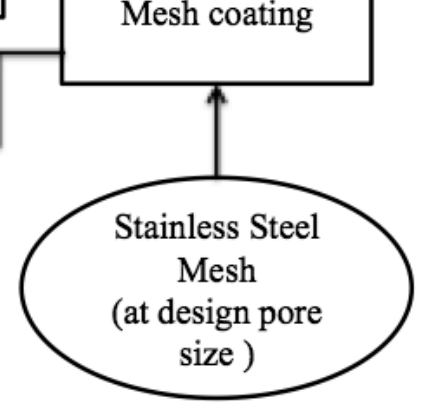

Figure 3. Experimental design flow sheet.

The dependent variables are separation time, percentage of water recovery and separation efficiency. As set of $4 \mathrm{X} 3$ factorial experiments with 3 points of repeatability are designed and conducted in which different sizes of mesh, and separation time, reclaimed oil and water recovery are varied.

\subsection{Characterization and Analysis}

\subsubsection{Water recovery and reclaimed oil}

After separation, water recovery and reclaimed oil were measured. The water recovery is the percentage of water filtered through the mesh compared to the original water content in the testing mixture by using equation (1). In equation 1 , $\left(V_{\text {water }}\right)_{\text {filtrate }}$ is the volume of the water present in the filtrate and $\left(V_{\text {water }}\right)_{0}$ is the volume of the water present in the initial feed mixture. The reclaimed oil is the percentage of the oil that did not pass through the coated mesh compared to the original oil percentage in the testing sample, which cab calculated equation (2), where $\left(V_{\text {oil }}\right)_{\text {filtrate }}$ is the volume of the oil present in the filtrate and $\left(V_{\text {oil }}\right)_{0}$ is the volume of the initial oil in the sample.

$$
\begin{gathered}
\text { Water Recovery }=\left(\frac{\left(V_{\text {water }}\right)_{\text {filtrate }}}{\left(V_{\text {water }}\right)_{0}}\right) * 100 \% \\
\text { Reclaimed Oil }=\left(1-\frac{\left(V_{\text {oil }}\right)_{\text {filtrate }}}{\left(V_{\text {oil }}\right)_{0}}\right) * 100 \%
\end{gathered}
$$

\subsubsection{Wettability}


To evaluate the performance of the coated mesh in oil/water separation, the under-water oil wettability of the hydrogel is investigated. The wettability of water and oil on the PAM-hydrogel coated mesh was obtained from contact angle (CA) measurements using Axisymmetric Drop Shape Analysis-Profile (ADSA-P). The oil wettability of the coated mesh was characterized by measuring the CAs of oil underwater with $5 \mu L$ droplet of 1,2-dichloroethane (DCE) as the probe liquid that is generated under-water on the coated mesh. The values are reported as the average of at least 4 drops per sample at different random locations. When oil droplets contacted with the PAM coated mesh, the image was captured at room temperature and then the contact angle measured using the ADSA software.

\subsubsection{Swelling Index Measurements}

The degree of ability of the hydrogel to hold water is calculated by using the equation (3).

$$
\text { Swelling ratio }=\frac{W_{s}-W_{d}}{w_{d}}
$$

Where, $\mathrm{W}_{\mathrm{s}}$ is the weight of the hydrogel in swollen state and $W_{d}$ is the weight of the hydrogel in dry state. Bulk hydrogel was used to study the swelling ratio, which was synthesised with the same polymer solution. Approximately $1 \mathrm{ml}$ of polymer solution was added to a petri dish and then exposed to UV light for 90 minutes until completely polymerized. The bulk polymer was rinsed with deionized water and then dried using a filter paper. The dried hydrogel is weighed in dry state and again after immersing in known amount of water for 5, 10, 20, 1440 and 2880 minutes. The swelling process was recorded by weighing the hydrogel in dry and swollen state.

\subsubsection{Oil Content in the Water Filtrate}

For controllable separation of oil and water mixtures, the visible oil existed in the permeated filtered water needs to be analyzed. The concentration of the oil after filtration which in turn affects the separation efficiency was estimated using a UV-VIS spectrophotometer according to a standard procedure. Initially, the absorption spectra of $5 \mathrm{~mL}$ of different oil concentrations (0.0$30.0 \mathrm{mg} / \mathrm{L}$ ) of oil solutions were recorded after diluting the oil in n-hexane as solvent in cuvettes. The solvent phase (n-hexane and oil) with unknown oil content was brought to the equipment for UV absorbance reading, which was determined under the same conditions used to obtain the calibration curves of oil in water. The concentration of oil was calculated by the intensity of the peak and the above-mentioned linear fit. At least two repetitions were made for determining the oil concentration in each sample. Oil concentration in the original sample was calculated by 
comparing the absorbance that obtained from the extracted sample to those that were prepared with known concentrations. The absorbance value was multiplied by a factor to give corresponding concentration, which is based on the Beer-Lambert law $(A=M C)$, where $A$ is the absorbance, $\mathrm{M}$ is the coefficient factor and $\mathrm{C}$ is the sample's concentration.

2.5.4 Morphology

The degree of uniformity of the hydrogel coating on the mesh is studied by obtaining images from scanning electron microscopy (SEM). Images were taken with an acceleration voltage from 5 to $10 \mathrm{kV}$. The test specimens, which were coated mesh $(0.5 \mathrm{~cm})$, were mounted on aluminum stubs with carbon tape. Before starting the measurements, samples were sputter coated with 10 $\mathrm{nm}$ of gold using a high-resolution sputter coater.

\subsubsection{Light Microscopy}

The droplet size of oil in oil/water mixture was obtained using the light microscopy (EVOS fl digital inverted microscope). Five images were taken randomly from different areas within the sample using a camera microscope. The corresponding diameter of the droplets was measured using Image $\mathrm{J} \circledast$ software.

\section{Results}

\subsection{Separation Efficiency}

Water recovery was calculated by using equation (1). For PAM hydrogel and two different concentrations of sodium acrylate in Na-Ac/AM copolymer, water recovery was in the range of 93 to $98 \%$ for all mesh sizes. Since the hydrogel holds some amount of water, the water recovery cannot exceed $98 \%$. Figure 4 shows the water recovery percentage for different mesh sizes (55, 65, 80 and $200 \mu \mathrm{m}$ ) based on different acrylamide monomer concentrations (50,55 and $60 \%$ ) in the PAM hydrogel-coated mesh and for two different concentrations of sodium acrylate in the Na-Ac/AM copolymer. Figures 4 a, and b illustrate the water recovery based on 5, and 10 (v/v) \% of original oil content in the mixture, respectively. It was observed that with an increase in the monomer concentration from 50 to $60 \%$, the separation time increases as the hydrogel gets thicker and also most of the samples that are collected do not have visible oil. A possible reason for this trend is due to the crosslink density. At lower AM concentrations, crosslink density will be more compared to higher AM concentrations which leads to the opposite trends for the water recovery vs. mesh size as observed in Figures 4a and 4b. Further, as the polymer concentration increases, viscosity of the solutions increases as well. It can be seen that the water recovery with 
the copolymer was less than that of polymer alone due to the higher swelling ability of the copolymer.

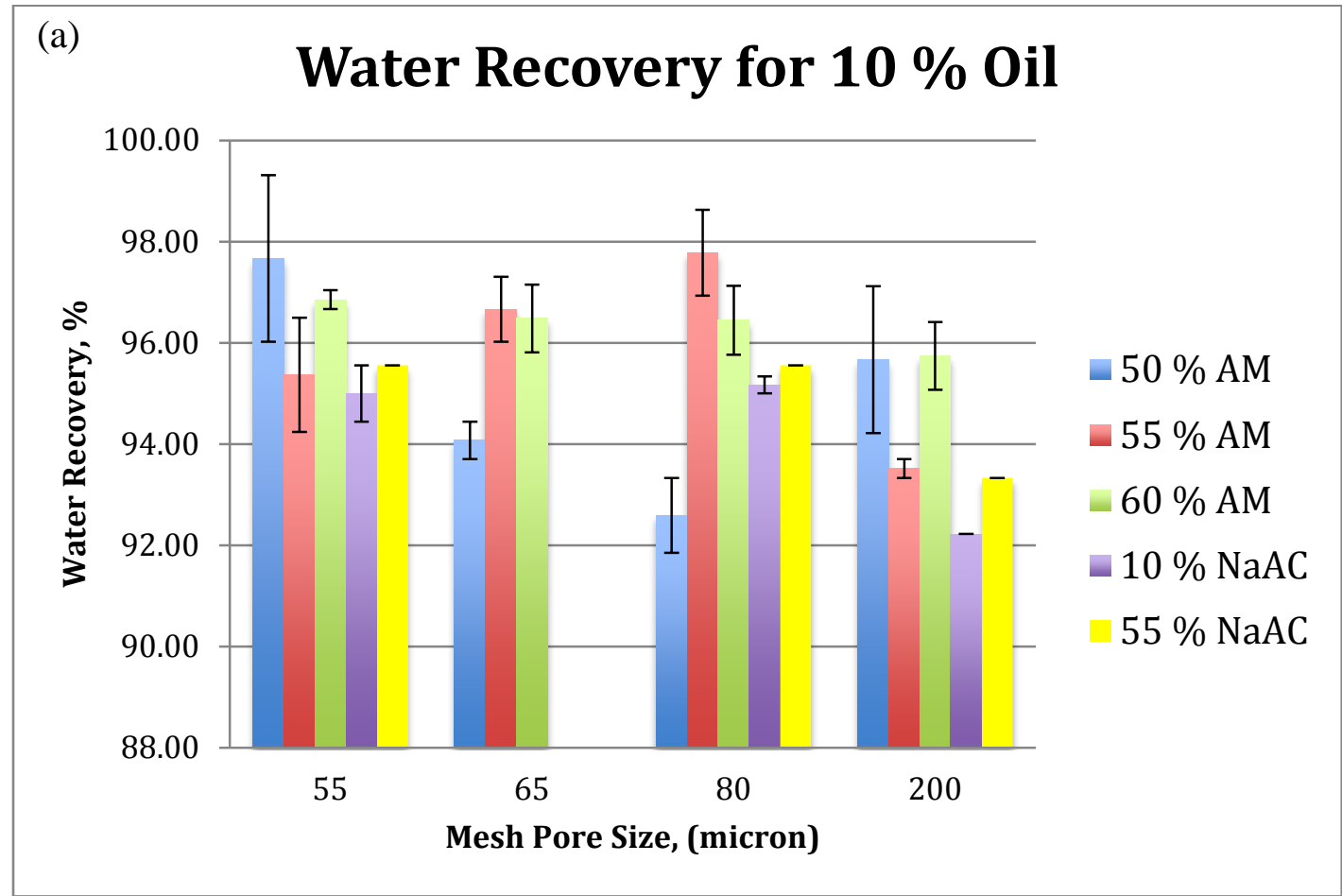

(b) Water Recovery for $5 \%$ Oil

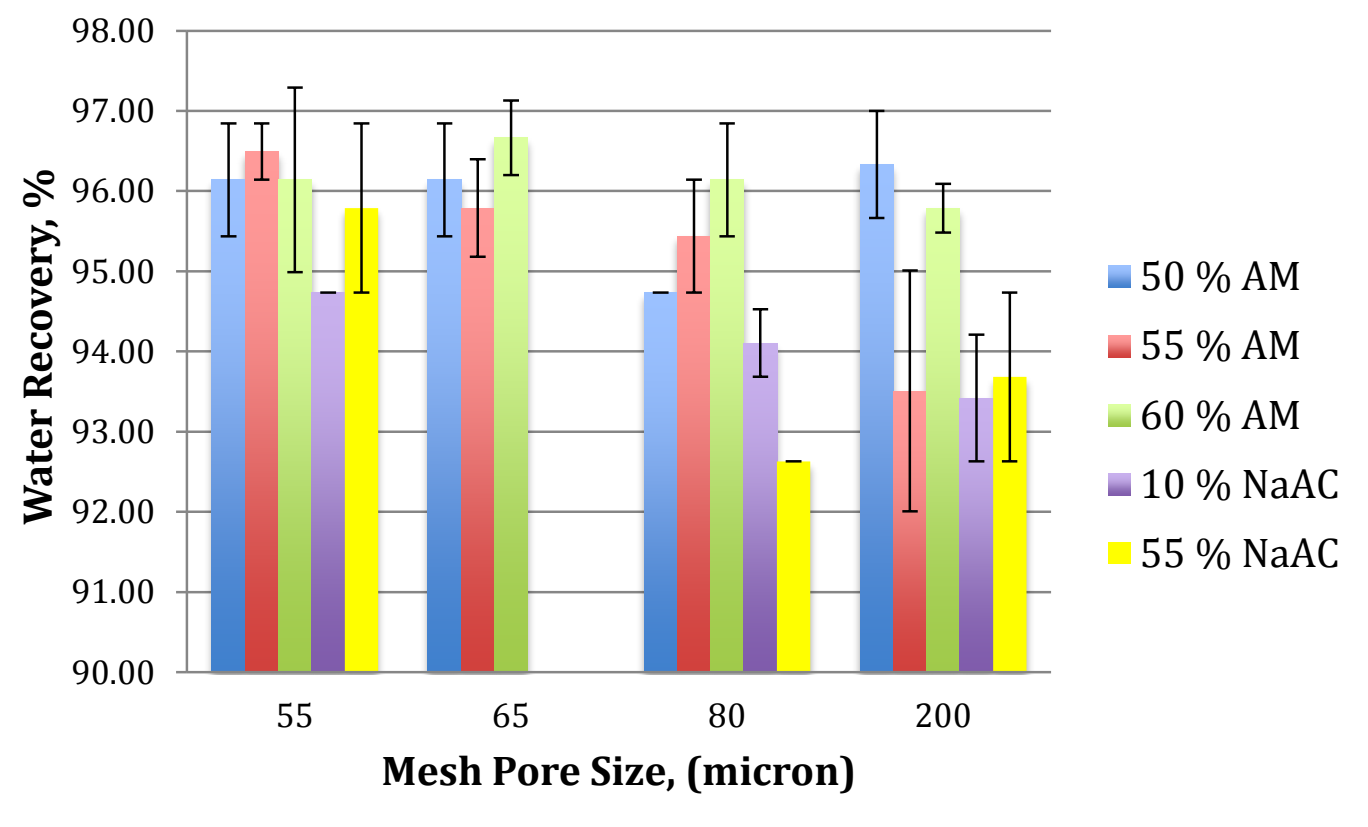


Figure 4. Water Recover Percentages of four different mesh (55, 65, 80 and 200 micron) based on 2 different AM concentrations in PAM polymer (50 and55 wt. \%) and 2 different Na-Ac in Na-AC/AM copolymer (10 and 55 wt. \%). A and B water recovery based on 5, and 10\% of original oil content in the mixture, respectively. Results are expressed as mean \pm standard deviation $(n=3)$.

\subsection{Reclaimed Oil}

The potential presence of residual oil in the filtrated water was measured with UV-visible spectroscopy. Figure 5 a, b and c shows the separation efficiency of PAM and Na-Ac/AM, for original oil \% in water which is 5,10 and $30 \%(\mathrm{v} / \mathrm{v})$ respectively. It can be seen that with an increase in the oil concentration in water, the separation efficiency decreased. Further, $30 \%$ oil in water was excluded from the investigation of the copolymer since higher oil concentration samples are separated using other techniques such as oil skimming, centrifuge, coagulation, floatation and sedimentation $[14,15]$. The performance of the tested hydrogel membranes is efficient at lower oil contents. Reclaimed oil \% was determined by measuring the oil percentage in the feed and in the corresponding filtrate (with UV-VIS spectrophotometer). As can be seen from Figure 5, the highest separation efficiency of Na-Ac/AM copolymer was for $5 \%$ oil and 10 \% NA-Ac in copolymer based on 200-micron mesh which corresponds to $93.8 \pm 0.24 \%$ (calculated using Equation (2)). Also, the separation efficiency of $55 \% \mathrm{Na}$-Ac was observed to be less than that of $10 \% \mathrm{Na}$-Ac due to the higher amount of sodium acrylate. Higher NaAc concentration will lead to higher swelling index which in turn increases the resistance for the water to pass through the hydrogel. 


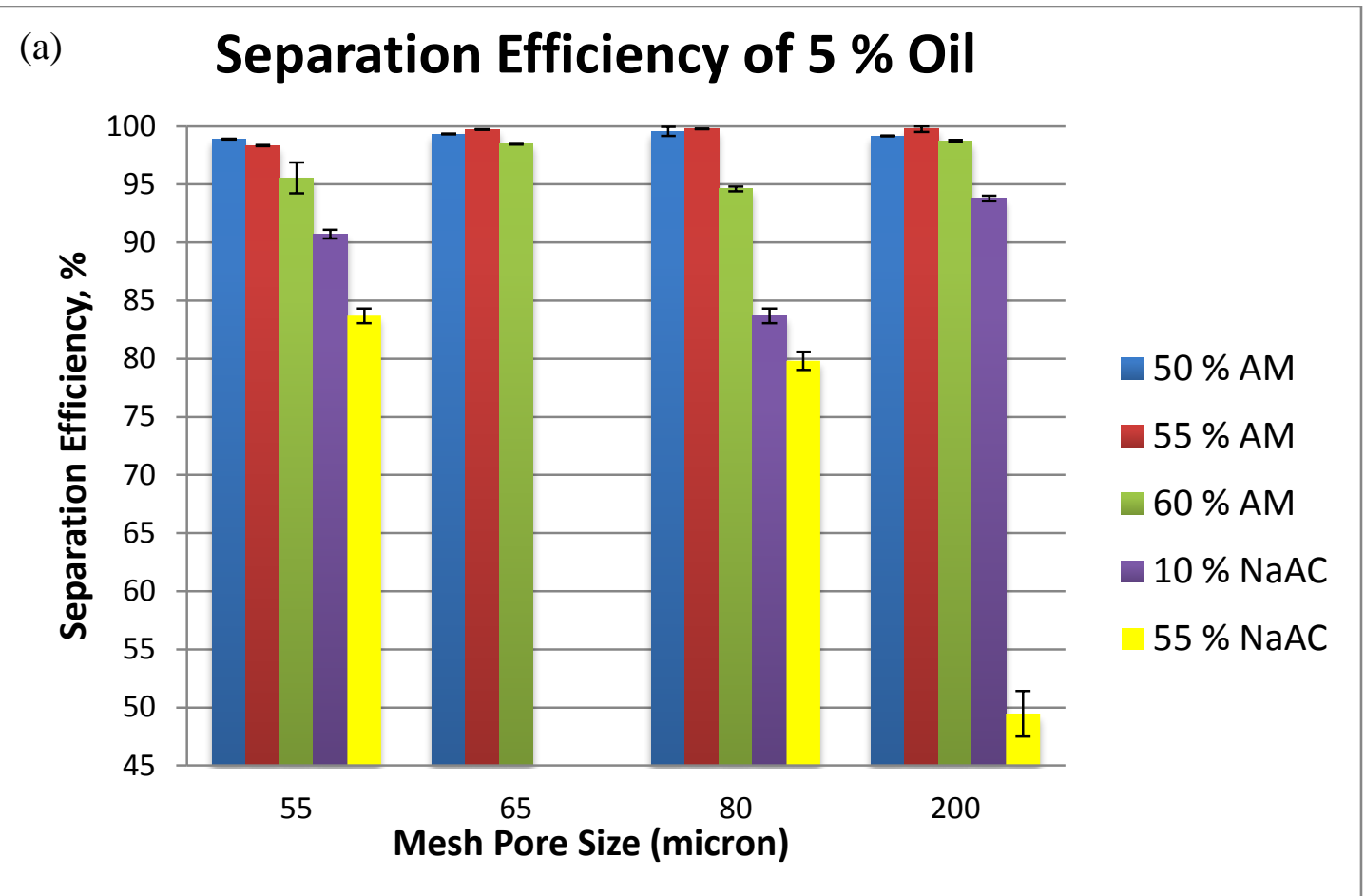

(b)

\section{Separation Efficiency of $10 \%$ Oil}

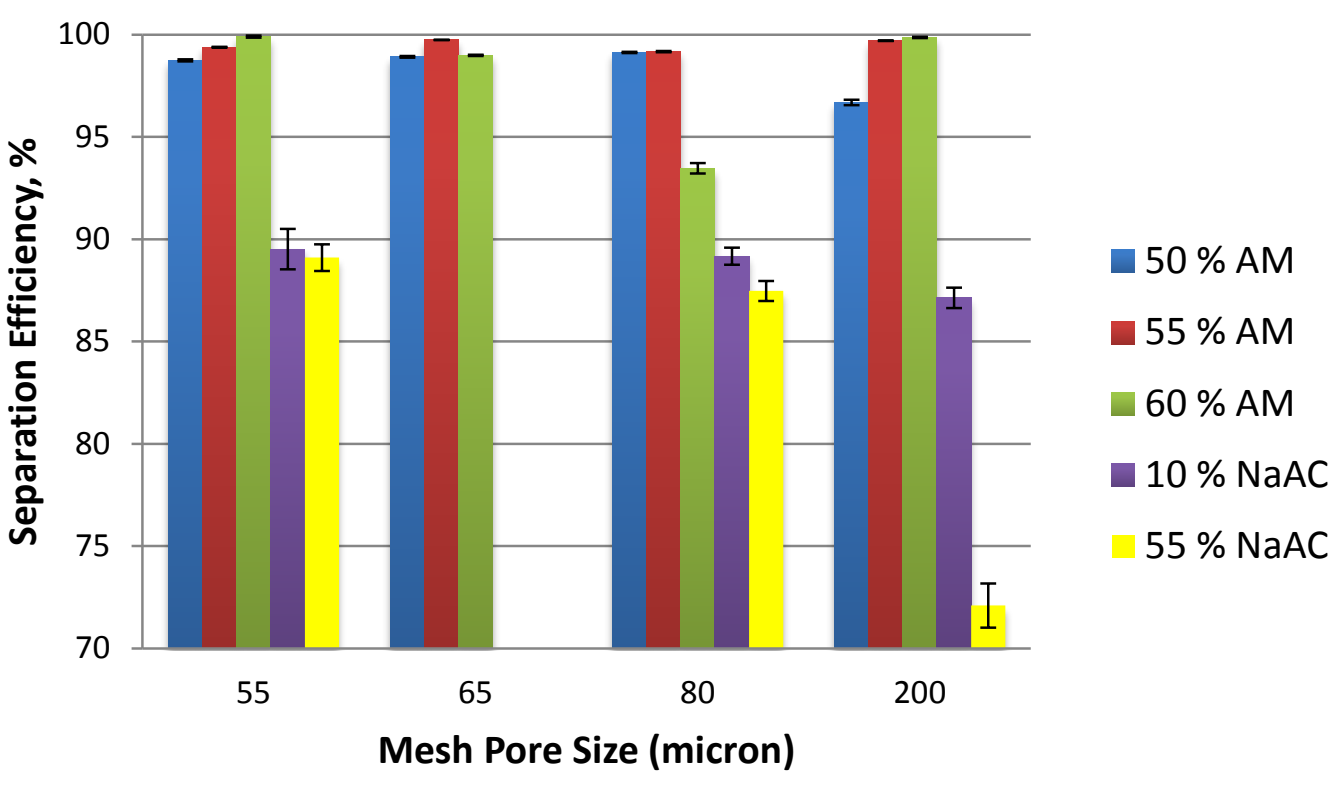

Figure 5. Separation Efficiency of PAM polymers and Na-AC/AM copolymer coated mesh based on different oil concentration (a). $5 \%$ oil, (b). $10 \%$ oil, Results are expressed as mean \pm standard deviation $(n=2)$. 
Due to the super-hydrophilic and the underwater oleo-phobic nature of the PAM coated mesh; water can easily wet and penetrate the treated sample while the oil is trapped on its surface. Figure 6 shows the separation results of $5 \%$ oil from oil/water mixture based on 55 micron size mesh for 55, and $60 \%$ of AM in PAM while Figure 7 shows separation results $10 \%$ oil from oil/water mixture based on 55 \% AM only in PAM. These samples showed separation efficiency of 98.3 and 95.6 and 99.4\%, respectively. Light microscopy study of the mixture before and after the separation process showed that there is a significant difference in the phase composition of the feed and the filtrate (as evident from Figure 6 (a), (c) and (e) and also from Figure 7 (a) and (b)). It is observed that the feed has much wider droplet size distribution, ranging from 11 to 98 $\mu m$ with higher frequency, which dropped from 40 to less than 5 and 17 after separation based on 55 and $60 \%$ AM, respectively (Figure 6 (b), (d) and (f)). This proved that the separation based on $55 \%$ AM was higher than $60 \%$ AM. This is due to increasing thickness of the hydrogel and higher swelling index in 60\% AM. The stability of the hydrogel is analysed by using the same hydrogel more than once for different oil/water mixtures. As shown in Figure 8, it is observed that the separation efficiency decreases (not very significantly) with an increase in the number repetitions of using the same hydrogel for separating oil from oil/water mixtures. This could be because of the change within the configuration of the crosslinked network when the hydrogel was dried to remove the trapped water. Further this should be confirmed from further advanced investigation.

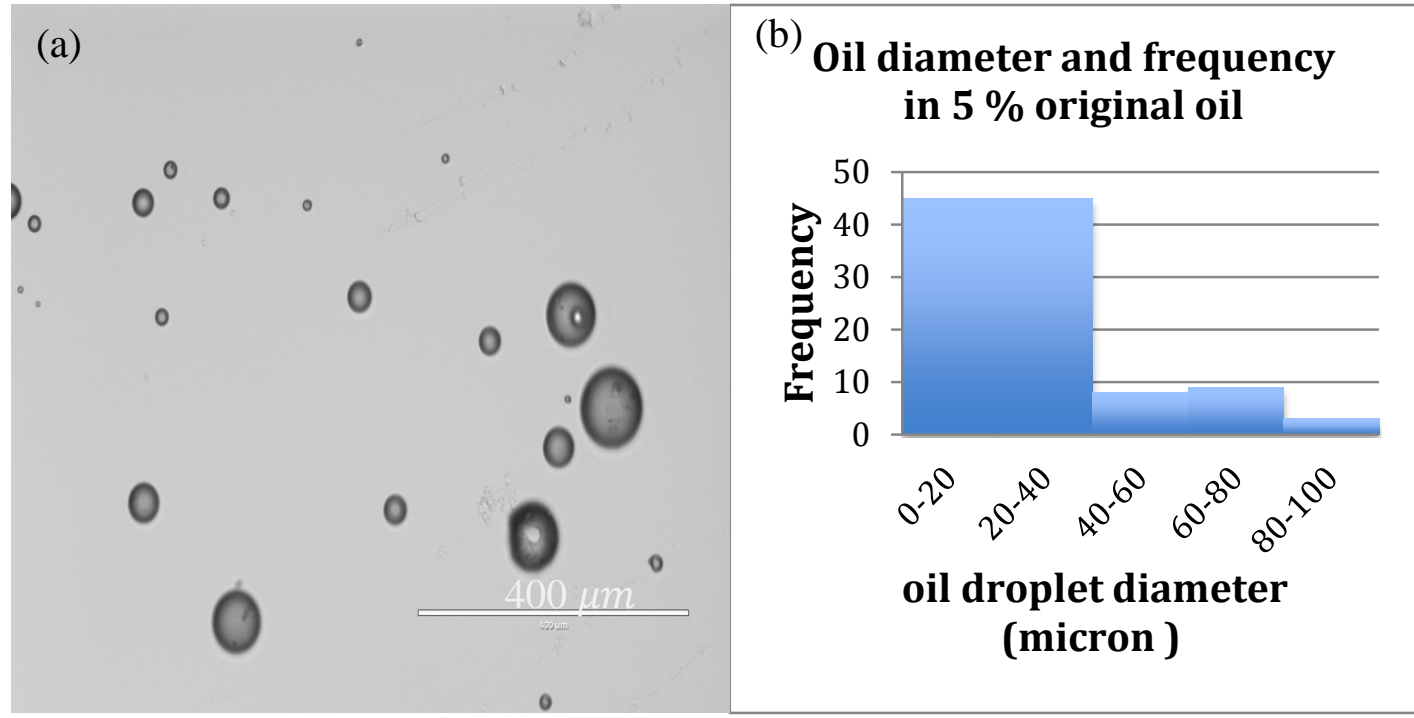

(c) 


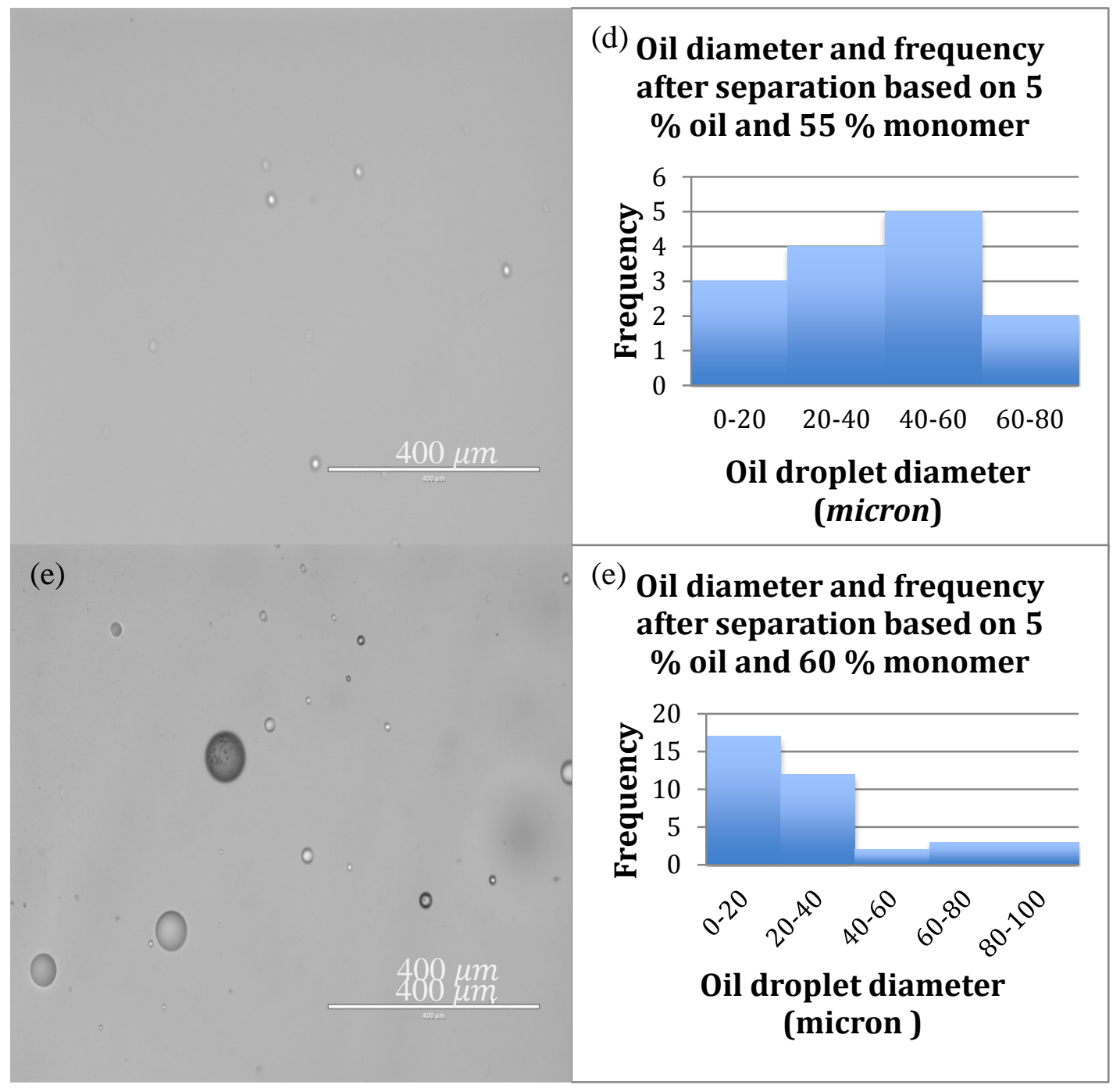

Figure 6. Oil/Water microscopic and oil size distribution before and after separation for separation of $5 \%$ original oil from water based on the 55-micron mesh. (a and b) Microscopic image and oil size distribution for feed composition of $5 \%$ oil. (c-e and d-f) are microscopic images and oil size distribution of filtrated water based on 55 and $60 \%$ AM in PAM, respectively.

(a)

(b) 


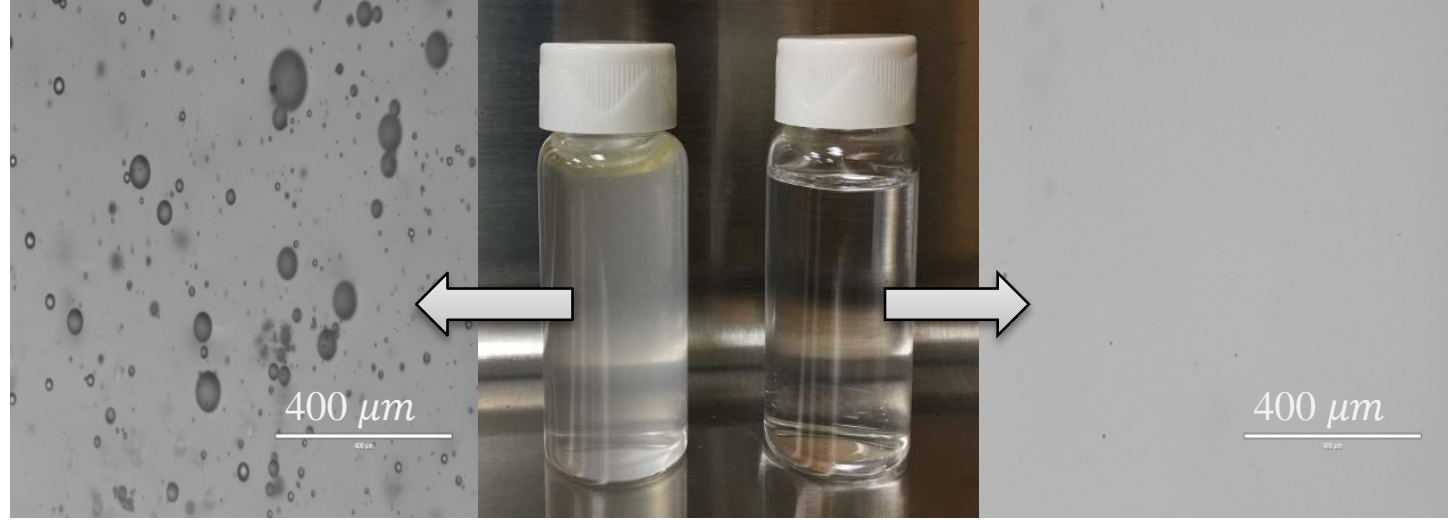

Figure 7 Microscopic and photographic images of $10 \%$ oil before and after separation, respectively. (A) is the $10 \%$ original oil, while (B) is after separation based on the 55 micron mesh and 55 \% AM in PAM polymer.

\subsection{Separation Time}

The effect of size of the mesh pore on the separation time is explained in this section. For a hydrogel coated mesh, it was observed that with an increase in the mesh pore size, the separation time decreased for 55, 65 and 80-micron sizes, while with 200 micron mesh has the longest separation time though it had a bigger pore size. With a 50\% AM monomer in the PAM hydrogel the separation time was the least. With an increase in the monomer concentration, the thickness of the membrane layer on the mesh increases which in turn decreases the separation time and makes the oil/water separation very slow.

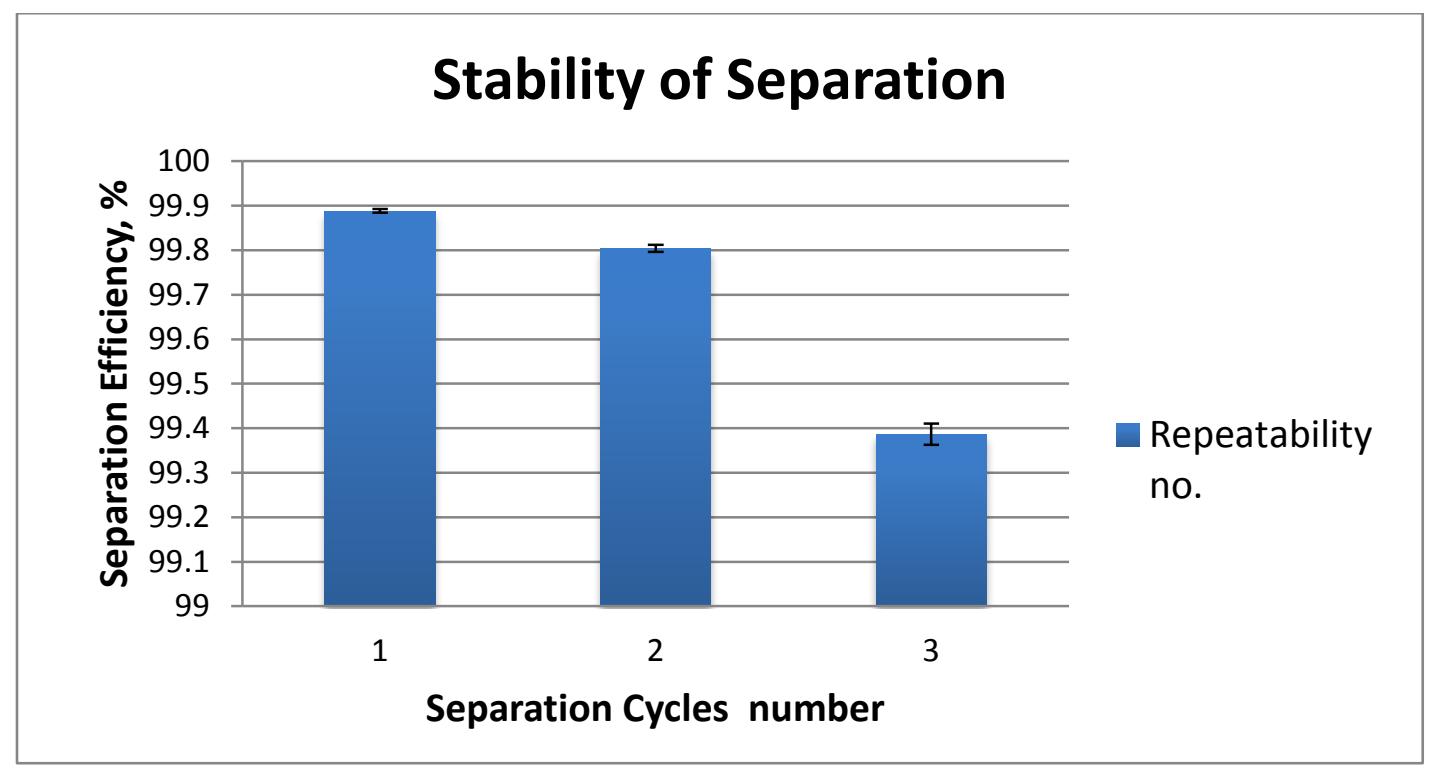

Figure 8. Separation stability $5 \%$ oil for PAM hydrogel coated mesh with $55 \%$ AM content 55 micron mesh size. Results are expressed as mean \pm standard deviation $(n=2)$. 
Figure 9 (a) and 9(b) illustrates the separation time of PAM coated mesh based on oil content in feed compositions for 5 and10 \% ( $/ / v)$, respectively. It is evident that as the initial oil concentration increased, the separation time decreased. Another important result observed in Figure 9(a) and 9(b) is that when the pore size is increased from 80 to 200, the separation time increased rather than decreasing. This was observed in all the experiments and shows a quadratic relationship between the separation time and pore size. Further this suggests that there is an optimum pore size after which separation time increases with an increase in the mesh pore size. Though increasing the mesh's pore sizes resulted in the decrease of the separation time, however, the separation efficiency decreased with a negative trend. Again this is an expected result where separation improves with finer mesh size. In conclusion, separation time was increased with increasing the monomer concentration in the polymer as it got thicker and decreased with increasing the oil content in water. However, for investigating the mesh pore sizes, it was found that separation time was decreased from 55 to 80 micron sizes, while it increased for the largest pore size, which was 200 micron. The separation time for the four different mesh sizes and three monomer concentrations were less than 30, 25 and 8 minutes based on 5, 10 and 30 oil \%, respectively.

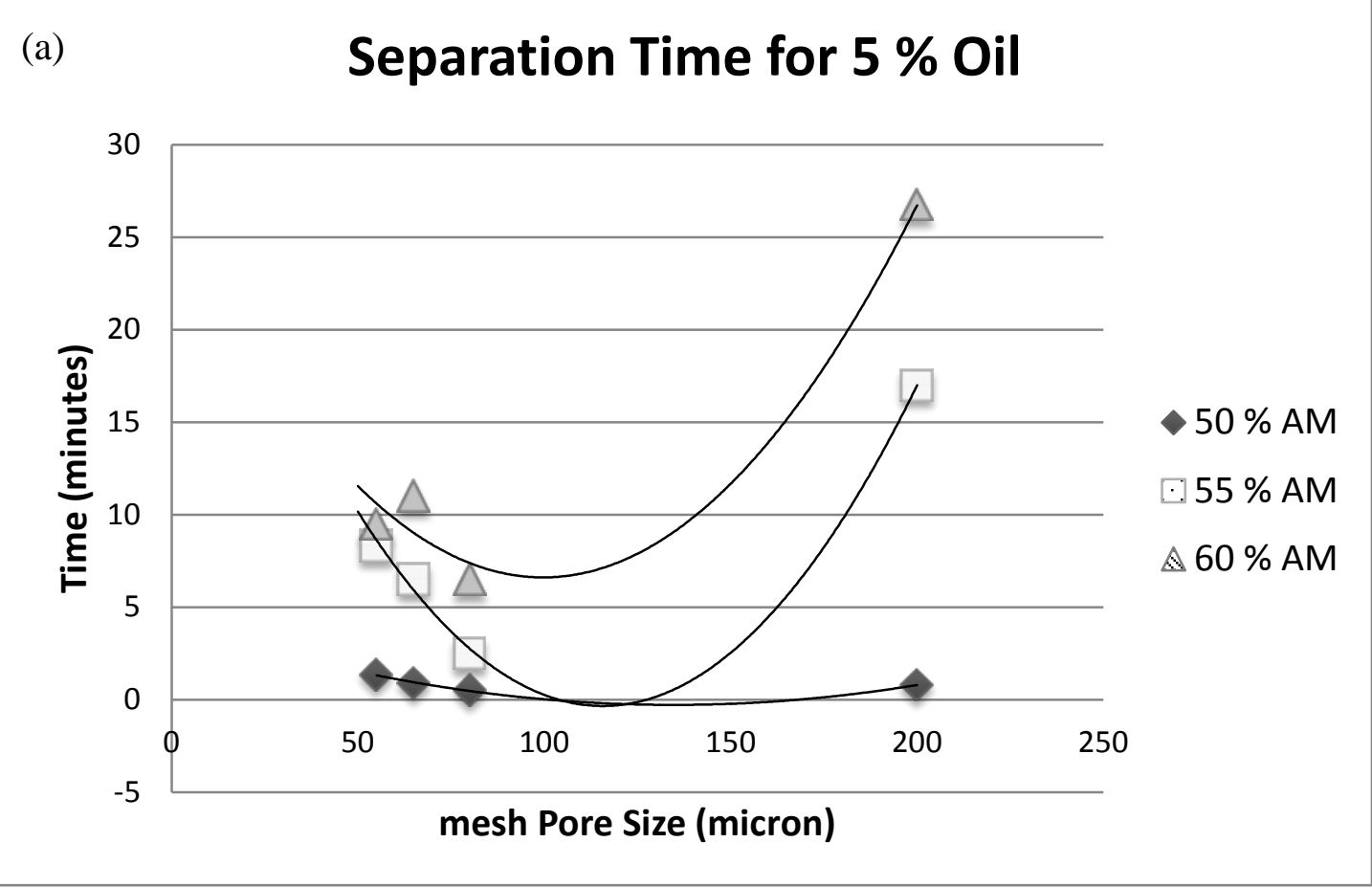




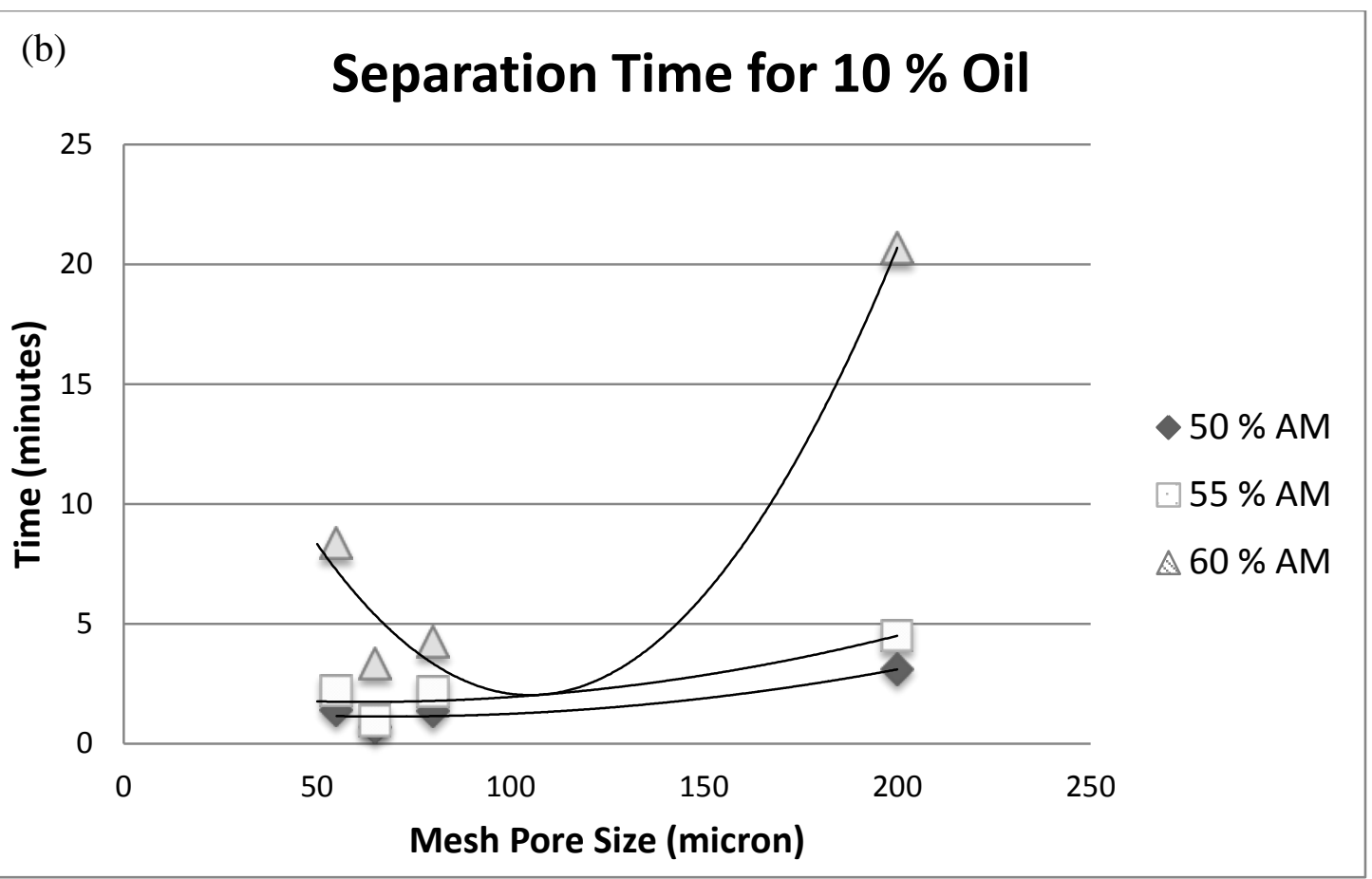

Figure 9. Separation time of oil/water mixture for PAM hydrogel coated mesh with three different acrylamide concentrations in the PAM, which are 50,55 and $60 \%$, based on 55,65,80 and 200 micron meshes. A andB separation time based on oil feed compositions 5 and 10\%. Results are expressed as mean \pm standard deviation $(n=3)$.

In the case of the hydrogels with the Na-Ac copolymer, separation time decreased significantly compared to the PAM hydrogel. Figure 10 (a) and (b) shows the separation time of oil/water mixture for Na-Ac/AM copolymer hydrogel with two different Na-Ac concentrations in the copolymer (10\% and $55 \%$ ) for three different mesh sizes (55, 80 and 200 micron mesh) based on $5 \%$ and $10 \%$ oil/water mixtures. Separation time of Na-Ac/AM copolymer was lower than with that of the PAM polymer. This is due to a higher swelling ratio of copolymer as it swells very fast than the PAM polymer which in turn is due to the ionic charges distributed evenly in the sodium acrylate based copolymer hydrogel. As the Na-Ac concentration increases the degree of swelling also increases. From Figure 10 (a) and (b), 55 \% Na-Ac hydrogel was faster than that of the $10 \% \mathrm{Na}$-Ac for the above reasons. Further, the separation time was less than 70 seconds compared to the several minutes it took for the membrane with PAM hydrogel alone. For the copolymer hydrogels also, the separation time showed a quadratic relationship with respect to the pore size. 


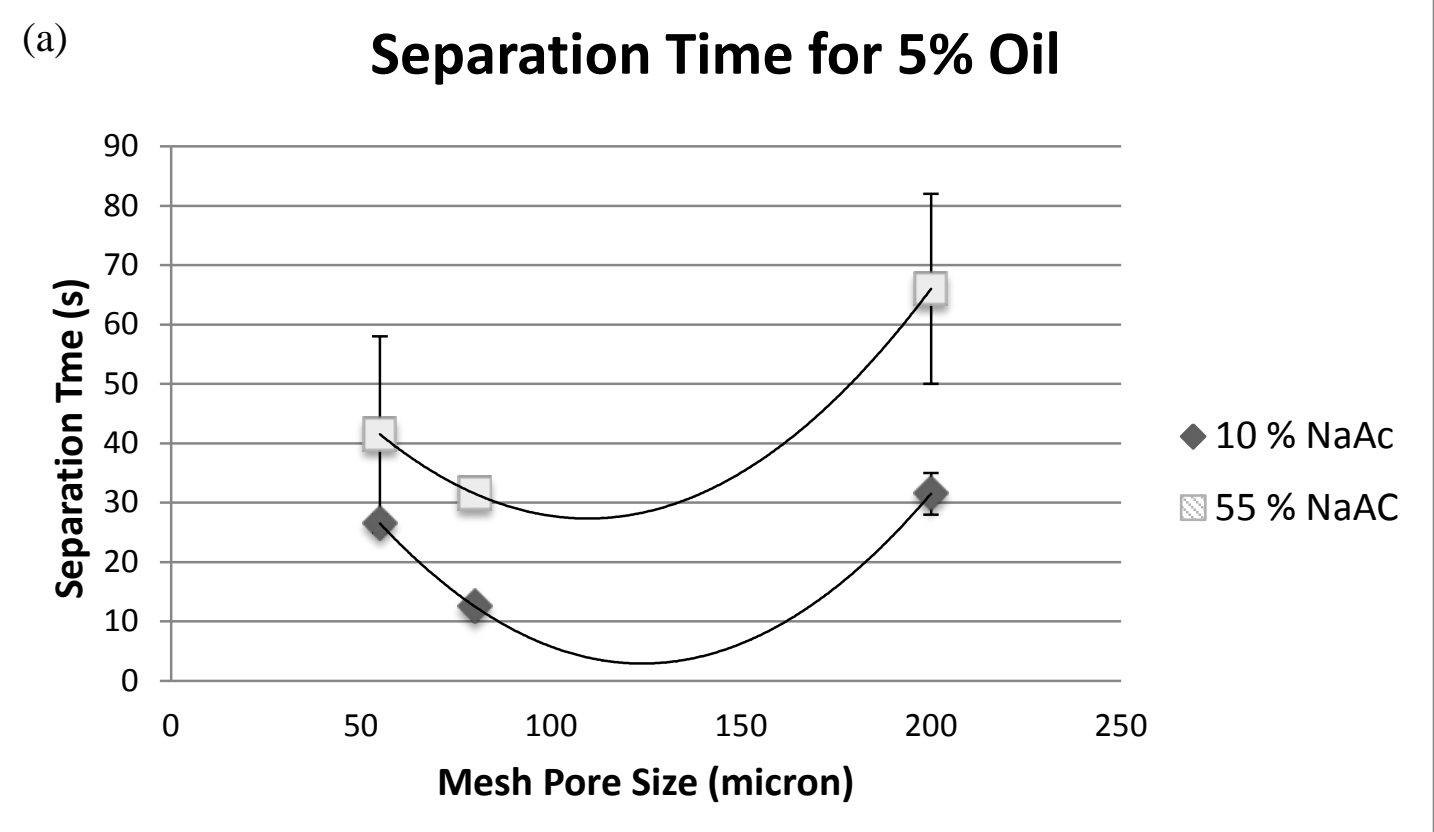

(b)

\section{Separation Time for $10 \%$ oil}

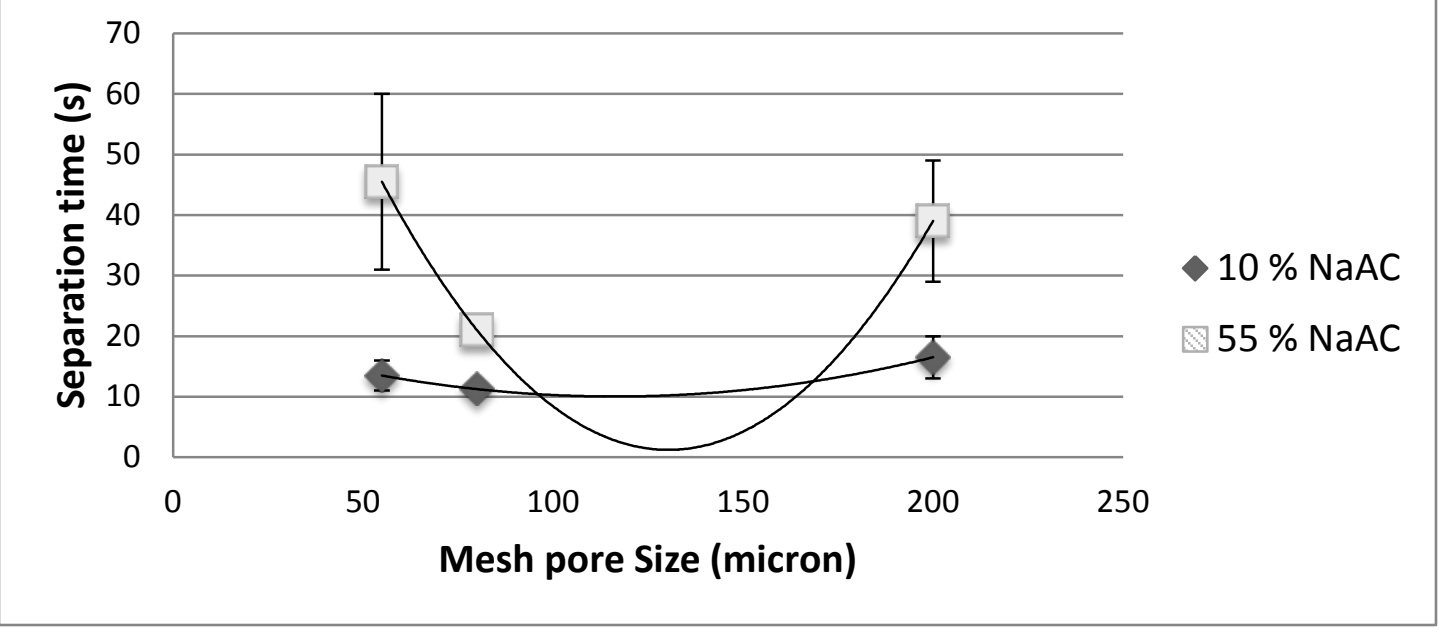

Figure 10. Effect of mesh size on separation time of oil/water mixture with Na-Ac/AM copolymer hydrogel for (a) 5\% oil/water mixture and (b) 10\% oil/water mixture. Results are expressed as mean \pm standard deviation $(\mathrm{n}=3)$.

\subsection{Wettability}

It is well known that the wettability of the solid surface mainly depends on both the geometrical structure (roughness) of the surface and the chemical compositions [16, 17]. The performance of the coated mesh is remarkable with extreme difference in the oil and the water contact angle. If underwater OCA is greater than OCA in air or greater than 150 degree, this will indicate the 
underwater super oleophobicity of hydrogel-coated mesh. This phenomenon is occurs due to the formation of water cushion between the solid surface of the PAM hydrogel coated mesh which is composed of water molecules in the rough surface and the oil droplet, which create a strong repulsive force due to interaction between the oil (non-polar) and the water (polar) molecules $[16,17]$. In air, water-wetting behavior on the PAM coated mesh was improved compared to the original uncoated mesh, which had a water CA of less than $5^{\circ}$. As shown in Figure 11, when water droplet $(5 \mu l)$ contacted with the surface of PAM (55 micron) coated mesh, it quickly spreads and penetrated through the mesh with a CA less than $5^{\circ}$, which indicates a good hydrophilicity and permeability of the coated mesh to water. Figure 12 shows the difference between water and oil droplet's shape in air on PAM coated mesh of 200-micron size. Water droplet was spread and permeated on the coated mesh, which was absorbed very fast (as shown in Figure 12 (a).), while in Figure 12 (b) oil droplet was not spread on the coated mesh with around 15 degrees. Therefore, PAM hydrogel coated mesh is super-hydrophilic in an air-solidliquid the three phase system with both oil contact angle (OCA) and water contact angle (CA) less than $10^{\circ}$.

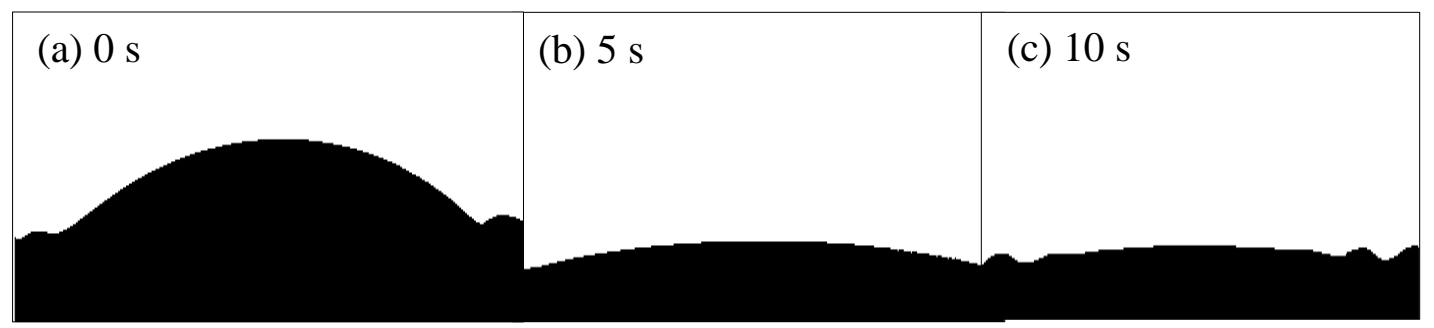

Figure 11. Water wettability of PAM hydrogel- coated mesh and spreading and permeating of water droplet $(5 \mu l)$. (a) at 0 seconds (b) at 5 seconds and (c) at 10 seconds.

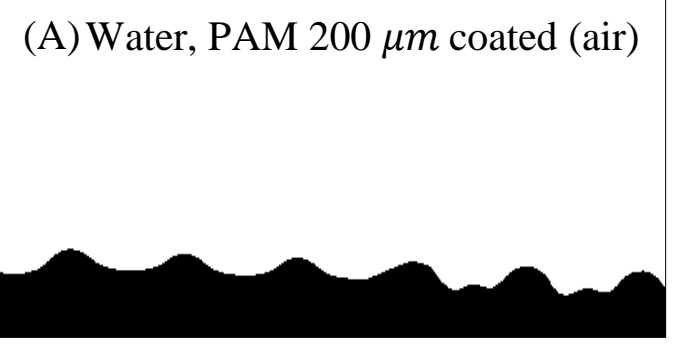

(B) Oil, PAM $200 \mu m$ coated (air)

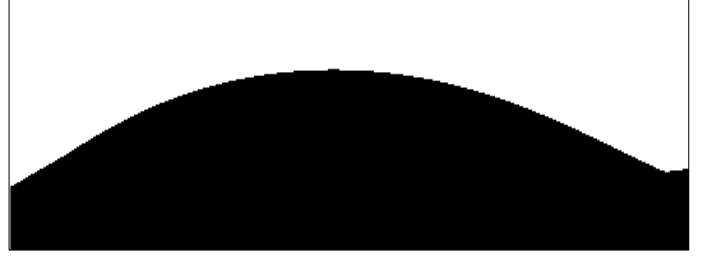

Figure 12. CA \& OCA based on 200 micron mesh in air PAM coated-mesh. (a) spreading and permeating water droplet on the coated mesh which was absorbed very fast; (b) Oil droplet on the coated mesh with 15 degree. 
Figure 13 shows the relationship between the pore diameter of the meshes and the under-water OCAs on the coating mesh with 3 different monomers. Within the experimental error, the OCAs for 55 micron mesh based on $60 \%$ AM in PAM was $140.03 \pm 0.88^{\circ}$. For 65 micron mesh it is $117.9 \pm 1.5^{\circ}$ after which it increases gradually with increasing pore size of the mesh. On the other hand, the water CAs on all the coated meshes was less than $5^{\circ}$. These results indicate that the hydrophobicity of the coating mesh is affected by the size of the pores and roughness of the surface. If the roughness of the membrane increases, then the contact area between the membrane and the oil decreases. Under-water, when the coated mesh contacts with the oil droplet, more water can be trapped in the rough microstructure, forming an oil/water/ solid composite interface. These trapped water molecules will also reduce the contact area between the oil and the coated mesh, resulting in large oil CA under-water, which leads to a dramatic increase in oleophobicity. For 60 \% acrylamide in the hydrogel, all the OCAs are greater than 115 degree and up to $140^{\circ}$ (as shown in Figure 13). The adhesion forces for different oils such as gasoline, vegetable oil, crude oil diesel, hexane and other petroleum is less than $5 \mu N$ on PAM hydrogel coated mesh [18], which proves that low adhesion properties can prevent PAM coated mesh from fouling by oil during the separation process.

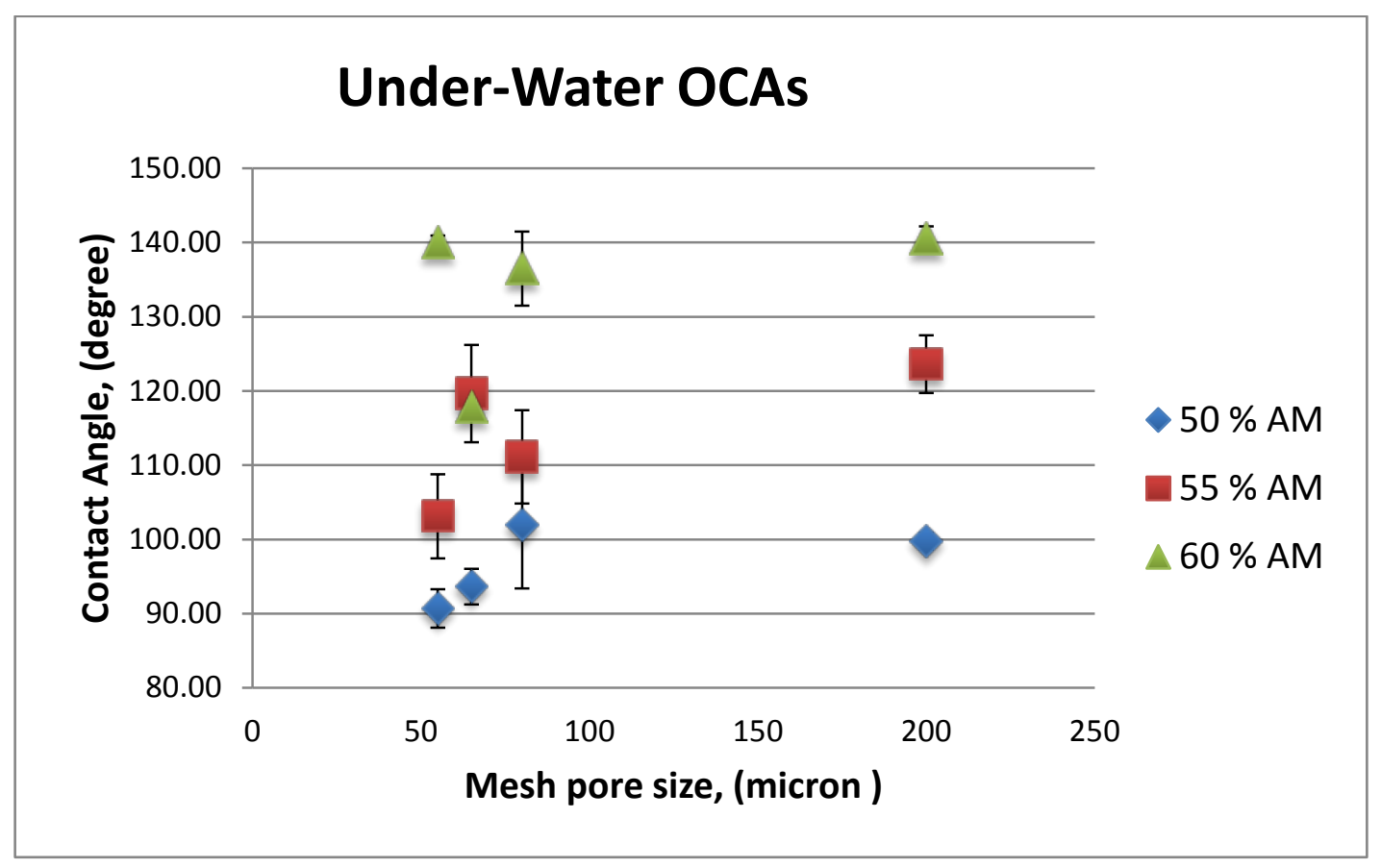


Figure 13. Effect of mesh size on the Under-water OCA for different monomer concentrations Results are expressed as mean \pm standard deviation $(n=4)$.

\subsection{Swelling Index}

Swelling Index was calculated to quantify the hydrogel ability to absorb water by using Equation (3). The swollen hydrogels were removed from water after 5, 10, 20, and 1440 and 2880 minutes, excess water was wiped with filter paper and then weighed quickly. Four samples were taken for each experiment, and the reported data points represent the averages of the four data points. Figure 14 shows that the swelling index of all monomer concentration increases gradually within 24 hours and remains almost constant until 48 hours. The swelling of the hydrogel is induced by the electronic repulsion of the ionic charges of its network [19]. The ionic charge content has a strong effect on the swelling. Sodium acrylate has many ionic units (-COONa), therefore, the swelling increases due to increasing anionic units [20]. The salt group is almost ionized, and a large number of hydrophilic groups appear. The hydrophilic group of AM/Na-Ac copolymers are higher than those of acrylamide, thus the swelling of AM/Na-Ac copolymers is greater than the swelling of PAM polymers. The 10 and $55 \%$ Na-Ac copolymers swell rapidly, and its swelling ratio was $272.15 \pm 0.29$ and $329.51 \pm 6.28 \%$ within 24 hours, respectively. The crosslinking concentration was maintained at $1.5 \%$ in PAM and the copolymer hydrogels. From the classical behavior of hydrogels, swelling ratio decreases with an increase in the crosslinking concentrations. This is due to the fact that the distance between the crosslink is smaller when the crosslinking amount increases, which in turn restricts its swelling capacity [21]. 


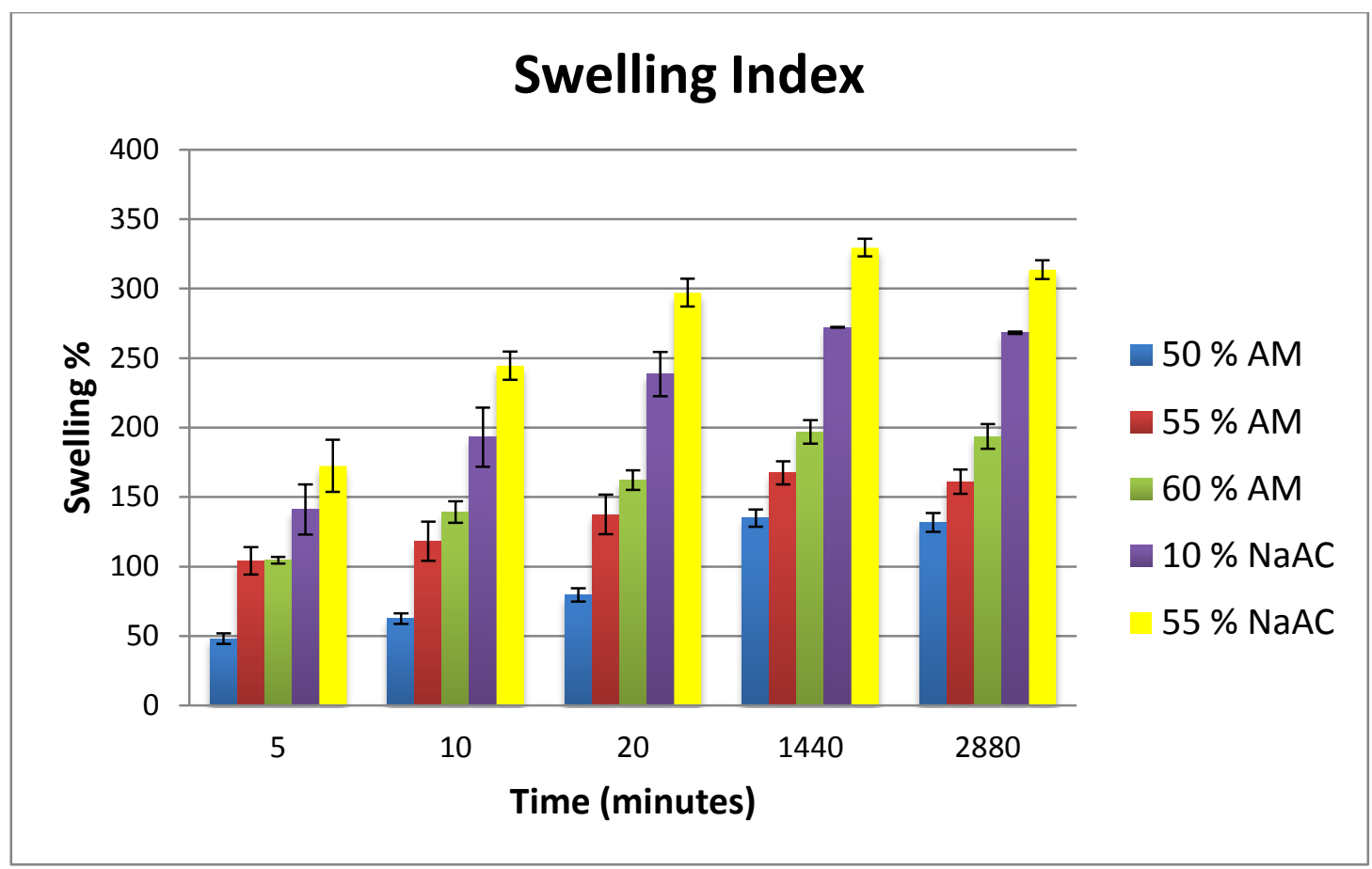

Figure 14. Swelling Index of 50, 55 and $60 \%$ monomer in PAM hydrogel, and 10 and $55 \%$ NaAc in the copolymer. Results are expressed as mean \pm standard deviation $(n=4)$.

\subsection{Membrane Morphology}

Scanning electron microscopy (SEM) imaging was carried out to characterize the surface morphologies of the mesh before and after coating. This will help to study the uniformness of the surface of the hydrogel membrane over the stainless steel mesh. More uniformness of the hydrogel over the steel mesh will have higher separation efficiency and less oil penetration through the membrane. Figures 15 (a) through (f) show the SEM images of PAM polymer (60 \%AM) coating on a stainless steel mesh. The micro-porosity of the coated mesh can be controlled by different mesh sizes and the amount of hydrogel. Figure 15 (a) shows the SEM image of the uncoated mesh with 200 micron size and Figure 15 (b) shows the same mesh coated with PAM hydrogel. Further, Figure 15 (c) and Figure (d) shows the large and enlarged view of the coated mesh. From these figures it is evident that the coating obtained after graft polymerization is uniform and almost all spacing among the pores of the mesh is completely filled with the hydrogel. Figure 15 (e) and Figure 15 (f) shows the corresponding SEM images with the Na-Am copolymer hydrogel. The copolymer hydrogel had less binding at the mesh joints compared to the PAM hydrogel (as can be seen in Figure 15 (e)) which could be the reason 
for observing more remnants of oil in the filtrate. It also shows large aggregation in the copolymer hydrogel with higher sodium acrylate, which held together by intermolecular hydrophobic association to form a 3D network and this is due to the high viscosity of the copolymer, which is in agreement with reported literature [22, 23]. Therefore, large aggregation shows strong intermolecular interaction and high swelling rate, where hydrogel was getting off the mesh which end up with open areas that tiny oil can pass through them.

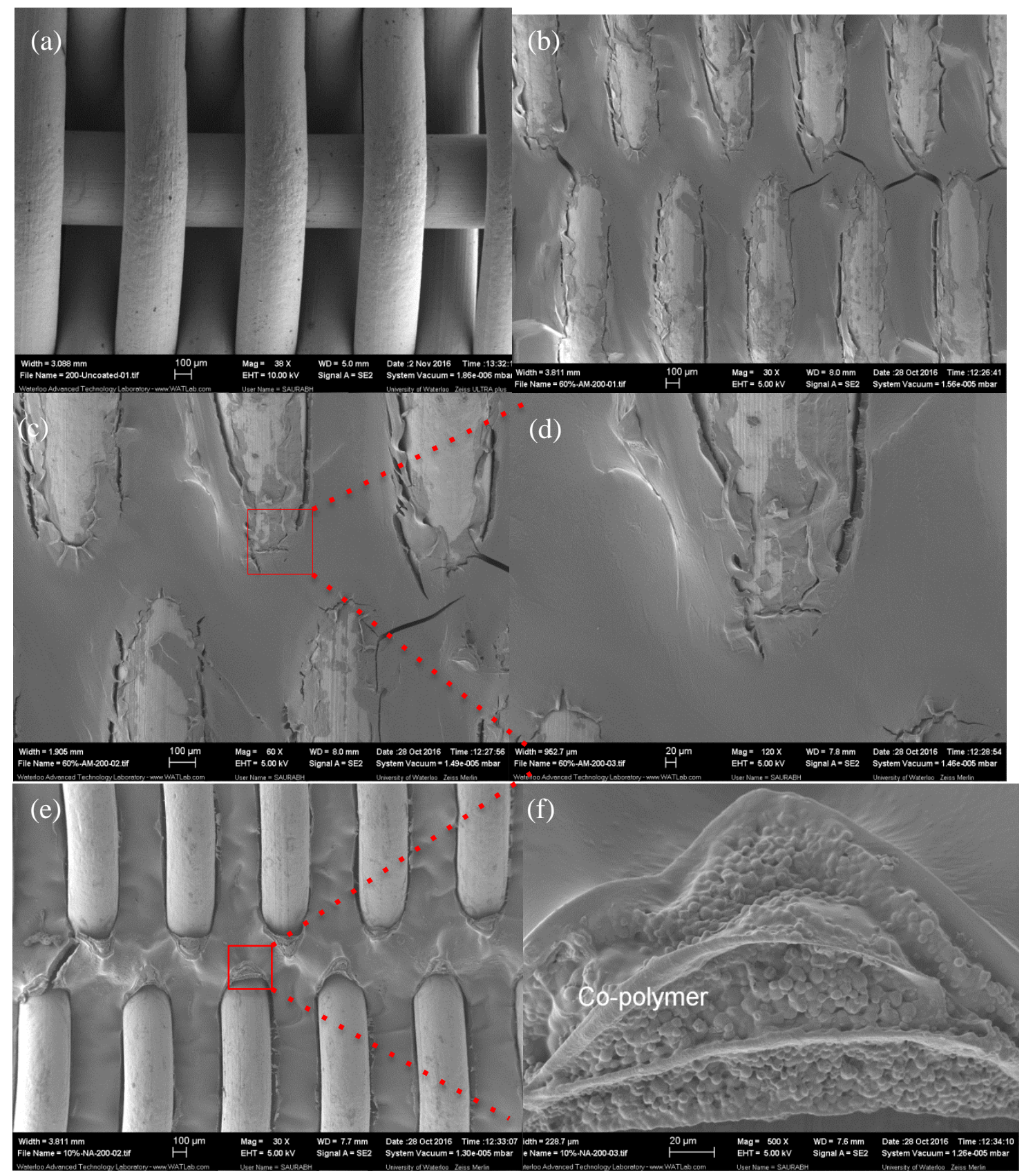


Figure 15. SEM images of 200 micron stainless steel mesh (a) uncoated (b) coated with PAM hydrogel (c) larger view (d) enlarged view (e) coated with Na-Ac copolymer hydrogel (f) enlarged view.

\section{Conclusions}

Reinforced hydrogels coated on stainless steel mesh was efficiently synthesized and used for oil water separations. Two different kinds of acrylamide based hydrogels were tested for separation efficiency. One of them is a PAM hydrogel and the other one is a copolymer of Na-Ac hydrogel. The effect of the mesh pore size and the composition of the hydrogel on the separation efficiency were evaluated. From the experimental results water recovery in the range of 93 to $98 \%$ was achieved. It was found that as oil concentration in water increases, the separation efficiency decreases. These reinforced hydrogels work effectively with lower concentrations of oil in water. Hydrogel with low monomer content formed thinner membrane than with a higher monomer concentration. The separation time is faster with the thinner membrane but separation efficiency is less than that of thicker hydrogel. In the hydrogels with the copolymer, very fine gaps were formed when it absorbs water through which some of the oil leaked into the filtrate. This problem can be overcome by choosing a different material for the reinforcement. The separation time decreased with an increase in the mesh size from 55 micro till 80 micron but increased when mesh size was 200 micron. A quadratic relationship was found between the mesh size and the separation time. Further, the separation time was lesser in the case of copolymer based hydrogel compared to the PAM hydrogel which is attributed to the fact that the extra ionic effect of the Na-Ac was having higher swelling index which allowed water to pass through the hydrogel quickly. 


\section{References}

1. Pichtel, J. Oil and Gas Production Wastewater: Soil Contamination and Pollution Prevention, Appl. Environ. Soil Sci. 1 - 24, 2016.

2. Ahmed, E. M. Hydrogel: Preparation, characterization, and applications: A review. J. Adv. Res. 6, 105-121, 2015.

3. Schmedlen, R. H., Masters, K. S. \& West, J. L. Photocrosslinkable polyvinyl alcohol hydrogels that can be modified with cell adhesion peptides for use in tissue engineering. Biomaterials 23, 4325-4332, 2002.

4. Hoare, T. R. \& Kohane, D. S. Hydrogels in drug delivery: Progress and challenges. Polymer 49, 1993-2007, 2008.

5. Atta, A. M., Ismail, H. S. \& Elsaaed, A. M. Application of anionic acrylamide-based hydrogels in the removal of heavy metals from waste water. J. Appl. Polym. Sci. 123, 2500-2510 , 2012.

6. Wen, Q., Di, J., Jiang, L., Yu, J. \& Xu, R. Zeolite-coated mesh film for efficient oil-water separation. Chem. Sci. 4, 591-595, 2013.

7. Mohana Raju, K. and Padmanabha Raju, M. Synthesis of novel superabsorbing copolymers for agricultural and horticultural applications. Polym. Int. 50, 946-951, 2001.

8. Zhou, M., Li Qian and Jiping, X. Study on acrylamide-sodium acrylate copolymer gels, Chin. J. Polym. Sci. 8, 1990.

9. Karadağ, E. \& Saraydin, D. Swelling of Superabsorbent Acrylamide/Sodium Acrylate Hydrogels Prepared Using Multifunctional Crosslinkers. Turk. J. Chem. 26, 863-876 (2002).

10. Lee, W.-F. \& Yuan, W.-Y. Thermoreversible hydrogels X: Synthesis and swelling behavior of the (N-isopropylacrylamide-co-sodium 2-acrylamido-2-methylpropyl sulfonate) copolymeric hydrogels. J. Appl. Polym. Sci. 77, 1760-1768, 2000.

11. Kalaleh, H.-A., Tally, M. \& Atassi, Y. Preparation of poly(sodium acrylate-co-acrylamide) superabsorbent copolymer via alkaline hydrolysis of acrylamide using microwave irradiation, Available: https://arxiv.org/ftp/arxiv/papers/1502/1502.03639.pdf, 2015.

12. Shukla, N. B. \& Madras, G. Photo, thermal, and ultrasonic degradation of EGDMAcrosslinked poly(acrylic acid-co-sodium acrylate-co-acrylamide) superabsorbents. J. Appl. Polym. Sci. 125, 630-639, 2012. 
13. Roger, P., Gérard, S., Burckbuchler, V., Renaudie, L. \& Judeinstein, P. Effect of the incorporation of a low amount of carbohydrate-containing monomer on the swelling properties of polyacrylamide hydrogels. Polymer 48, 7539-7545, 2007.

14. Dickhout, J., J. Moreno, P. Biesheuvel, L. Boels, R. Lammertink, and W. de Vos, Produced water treatment by membranes: A review from a colloidal perspective. Journal of colloid and interface science, 487, 523-534, 2017.

15. Zhang, F., S. Gao, Y. Zhu, and J. Jin, Alkaline-induced superhydrophilic/underwater superoleophobic polyacrylonitrile membranes with ultralow oil-adhesion for high-efficient oil/water separation. Journal of Membrane Science, 513, 67-73 2016.

16. Wen, Q., Di, J., Jiang, L., Yu, J. \& Xu, R. Zeolite-coated mesh film for efficient oil-water separation. Chem. Sci. 4, 591-595, 2013.

17. Zhang, L., Zhang, Z. \& Wang, P. Smart surfaces with switchable superoleophilicity and superoleophobicity in aqueous media: toward controllable oil/water separation. NPG Asia Mater. 4, e8, 2012.

18. Ding, C. et al. PANI nanowire film with underwater superoleophobicity and potentialmodulated tunable adhesion for no loss oil droplet transport. Soft Matter 8, 9064-9068, 2012.

19. Karadağ, E. \& Saraydin, D. Swelling of Superabsorbent Acrylamide/Sodium Acrylate Hydrogels Prepared Using Multifunctional Crosslinkers. Turk. J. Chem. 26, 863-876, 2002.

20. Yao, K.-J. \& Zhou, W.-J. Synthesis and water absorbency of the copolymer of acrylamide with anionic monomers. J. Appl. Polym. Sci. 53, 1533-1538, 1994.

21. Roger, P., Gérard, S., Burckbuchler, V., Renaudie, L. \& Judeinstein, P. Effect of the incorporation of a low amount of carbohydrate-containing monomer on the swelling properties of polyacrylamide hydrogels. Polymer 48, 7539-7545, 2007.

22. Kang, W. et al. Solution behavior of two novel anionic polyacrylamide copolymers hydrophobically modified with $n$-benzyl- $n$-octylacrylamide. Polym. Eng. Sci. 52, 2688-2694 2012.

23. Bin, Z., Hu, H., Chen, M. \& Liu, W. Synthesis of associating poly(acrylic acid) in supercritical carbon dioxide and its solution properties. Colloid Polym. Sci. 282, 1228-1235 2004. 